\title{
Author Correction: Hydrophilic microporous membranes for selective ion separation and flow-battery energy storage
}

Rui Tan, Anqi Wang (D), Richard Malpass-Evans, Rhodri Williams, Evan Wenbo Zhao, Tao Liu, Chunchun Ye, Xiaoqun Zhou, Barbara Primera Darwich, Zhiyu Fan, Lukas Turcani (D), Edward Jackson (D), Linjiang Chen, Samantha Y. Chong (D), Tao Li, Kim E. Jelfs, Andrew I. Cooper, Nigel P. Brandon, Clare P. Grey, Neil B. McKeown (D) and Qilei Song (D)

Correction to: Nature Materials https://doi.org/10.1038/s41563-019-0536-8, published online 2 December 2019.

In the version of this Article originally published, Rhodri Williams was missing from the author list; he should have been listed after Richard Malpass-Evans with the affiliation EaStChem School of Chemistry, University of Edinburgh, Edinburgh, UK. The text 'R.W. devised and synthesized PIM-BzMA-TB.' has also been added to the author contributions section after 'R.M. synthesized TB-PIMs and carried out characterizations.'

Published online: 22 December 2019

https://doi.org/10.1038/s41563-019-0593-z

(c) The Author(s), under exclusive licence to Springer Nature Limited 2019 